(C) 2020 IEEE. Personal use of this material is permitted. Permission from IEEE must be obtained for all other uses, in any current or future media, including reprinting/republishing this material for advertising or promotional purposes,creating new collective works, for resale or redistribution to servers or lists, or reuse of any copyrighted component of this work in other works.

\title{
Multi-GPU Parallelization of the NAS Multi-Zone Parallel Benchmarks
}

\author{
Marc González and Enric Morancho
}

\begin{abstract}
GPU-based computing systems have become a widely accepted solution for the high-performance-computing (HPC) domain. GPUs have shown highly competitive performance-per-watt ratios and can exploit an astonishing level of parallelism. However, exploiting the peak performance of such devices is a challenge, mainly due to the combination of two essential aspects of multi-GPU execution. On one hand, the workload should be distributed evenly among the GPUs. On the other hand, communications between GPU devices are costly and should be minimized. Therefore, a trade-of between work-distribution schemes and communication overheads will condition the overall performance of parallel applications run on multi-GPU systems.

In this paper we present a multi-GPU implementation of NAS Multi-Zone Parallel Benchmarks (which execution alternate communication and computational phases). We propose several work-distribution strategies that try to evenly distribute the workload among the GPUs. Our evaluations show that performance is highly sensitive to this distribution strategy, as the the communication phases of the applications are heavily affected by the work-distribution schemes applied in computational phases. In particular, we consider Static, Dynamic and Guided schedulers to find a trade-off between both phases to maximize the overall performance. In addition, we compare those schedulers with an optimal scheduler computed offline using IBM CPLEX.

On an evaluation environment composed of 2 x IBM Power9 8335-GTH and 4 x GPU NVIDIA V100 (Volta), our multi-GPU parallelization outperforms single-GPU execution from 1.48x to 1.86x (2 GPUs) and from 1.75x to 3.54x (4 GPUs). This paper analyses these improvements in terms of the relationship between the computational and communication phases of the applications as the number of GPUs is increased. We prove that Guided schedulers perform at similar level as optimal schedulers.
\end{abstract}

Index Terms-Multi-GPU Parallelization, Load Balancing, Static, Dynamic, Guided Schedulings

\section{INTRODUCTION}

G PU-based computing systems have been widely acJ cepted as a general solution for the High Performance Computing (HPC) community. Many computing domains have incorporated their usage though being necessary significant changes to adapt applications to such new systems. In general, the efforts to redesign, implement and tune an application to run on GPU-based systems are worth it due to the impressive performance levels that GPU-based systems can deliver. Machine Learning, BioInformatics, Scientific Computing and many more are domains that have clear examples of representative applications like TensorFlow [1], Caffe [2], Smith-Waterman [3], Alya [4] that recently developed the support for GPU-based systems.

The efforts to target a GPU-based system are related to essential programmability aspects of GPUs. Programmers have to modify their application with new constructs embedded in high level languages (e.g.: CUDA in C++ or Fortran), or with language extensions in the form of compiler directives (e.g.: OpenCL). Besides, programmers have to redefine their application design according to architectural features that GPU-based systems impose: the separation of the memory address space in several subspaces, manual memory allocation per each GPU, and intensive code tuning to achieve the optimal mapping between work units and GPU threads (e.g.: keep the appropriate relation between

- The authors are with the Computer Architecture Department, Universitat Politècnica de Catalunya - Barcelona Tech, Spain.

E-mail: \{marc,enricm\}@ac.upc.edu thread computation and register usage). For single-GPU parallelization, fine-grain parallelism is mapped to the GPU in the form of kernel invocations. In contrast, multi-GPU parallelization requires the exploitation of a coarser level of parallelism. This parallelism, orchestrated from one or more CPUs, requires the definition of memory-allocation strategies for each GPU, work-distribution schemes to balance the work assigned to each GPU, and communication phases whenever is necessary to exchange values between GPUs. For the latest aspect, multi-GPU systems are limited by the network topology that connects the GPUs. In general, GPU communications depend on the memory footprint induced by the work-distribution schemes. Therefore, these decisions will inherently affect the communication patterns. A trade-off exists between the work-distribution schemes and their impact on communication phases. Peer-to-peer data transfers should be exploited and GPU-to-CPU, CPUto-GPU data transfers should be avoided to minimize their extra communication overhead (e.g.: usage of CPU memory to communicate a pair of GPUs). To sum up, multi-GPU parallelization poses the challenges of exploiting two levels of parallelism: a fine-grain level exploited in the form of GPU kernels plus a coarser level exploited by the parallel execution on several GPUs. Besides, the parallelization has to distribute the workload considering at the same time load balancing and communication overheads.

Specifically for the scientific computing domain, the NAS Parallel Benchmarks (NPB) [5] and their multi-zone variation (NPB-MZ) [6] correspond to standard benchmark suites that allow studying the previous aspects within several numerical applications. There are implementations 
for distributed execution (e.g: using the Message Passing Interface (MPI)), for the shared memory standard OpenMP and for GPU-based systems (e.g.: OpenACC and CUDA). Currently, their GPU versions are limited to the usage of a single GPU, not being able to support multi-GPU execution.

The main contribution of this paper is the implementation of a multi-GPU parallelization of the NPB-MZ benchmark suite. The proposal defines a multi-level parallelization where fine-grain and coarse-grain parallelism are combined. Due to the nature of NPB-MZ benchmarks (they alternate Computation and Communication phases), the workload-distribution strategy heavily influences on the communication patterns between the GPUs. Consequently, we have considered several workload-distribution strategies (static, dynamic and guided schedulers). The proposal has been implemented with CUDA 10.0 and evaluated on a IBM Power9 8335-GTG and 4 GPUs NVIDIA V100 (Volta). The evaluation shows that Guided schedulers beat all the other schedulers in finding a trade-off between the execution of both phases. Our implementation outperforms single-GPU implementation by from $1.48 \mathrm{x}$ to $1.86 \mathrm{x}$ (2 GPUs) and from $1.75 x$ to $3.54 x$ (4 GPUs), depending on the application and the trade-off between both phases.

This paper is organized in the following sections: Section 2 describes the sources and nature of the parallelism in the NPB-MZ suite, Section 3 details the trade-off between the work scheduler and the communications costs, Section 4 evaluates our implementation, Section 5 discusses the works related to this paper and, finally, Section 6 concludes the paper with its main conclusions.

\section{Benchmark Characterization}

\subsection{NAS-MZ Benchmarks}

The Multi-Zone NAS Parallel Benchmarks (NPB-MZ) [6] are based on a very well-known and studied application suite, the NAS Parallel Benchmarks (NPB) [5]. They have been widely used for testing the capabilities of parallel systems, being able to expose many computation aspects through performance characterization, from low-level architectural details up to the evaluation of runtime parallel libraries and parallelization tools (e.g.: OpenCL, OpenMP and CUDA). The applications contained in the suite exhibit both coarseand fine-grain parallelism and all of them implement iterative methods, repeating a pattern where computational phases alternate with communication phases.

The applications in the NPB-MZ suite are: Lower-Upper Symmetric Gauss-Seidel (LU-MZ), Scalar Penta-diagonal (SP-MZ), and Block Tri-diagonal (BT-MZ). All of them solve discretized versions of the unsteady, compressible NavierStokes equations in three spatial dimensions.

The suite defines several problem sizes identified as input classes S, W, A, B, C, and D (from smaller to greater sizes). For all of them, verification values are given in order to test the correctness of the execution.

\subsection{Zone definition}

Zones are organized into a 2D-grid. Each zone has 4 adjacent zones (north, south, east and west). For instance, zone A in Fig. 1 is adjacent to its 4 darkened neighboring zones. For zones at the grid edges, adjacency assumes a torus topology. For instance, north adjacent zone of zone B is on the other side of its column. Analogously, east adjacent zone of zone $\mathrm{C}$ is placed on the other side of its row. Zone adjacency will be taken into account through this work because to completely update a zone, some information (boundary values) is required from its adjacent zones.

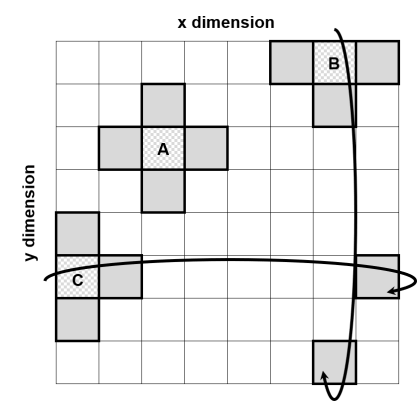

Fig. 1. Adjacency definition within the 2D-grid where zones are embedded. Zones have 4 adjacent zones in the north, south, east and west directions. Zones at the grid edges have a torus-like adjacency.

Through this work zones will be identified by an integer value (zone-id) that ranges from 0 to the total number of zones minus one. zone-id is analogous to the row-major order of the 2D-grid where zones are embedded. That is, in Fig. 1, zone number 0 corresponds to the top-left zone, zone number 7 corresponds to the top-right zone, zone number 56 corresponds to the bottom-left zone and zone number 63 corresponds to the bottom-right zone.

NPB-MZ applications operate over a main data structure organized as a 3D mesh. The mesh is sliced across both $\mathrm{x}$ and y dimensions into cuts (equispaced in both LU-MZ and SP$\mathrm{MZ}$, non equispaced in BT-MZ); these slices are referred as zones. Each zone is represented by a set of matrices whose sizes depend on both the benchmark and the input class. TABLE 1 shows, for each input class and benchmark, the number of zones that compose the 3D mesh and the number of time-step iterations performed by the numerical method; also, it shows the total mesh size and the amount of memory that must be allocated for each input class.

\begin{tabular}{|c|c|c|c|c|c|c|c|c|}
\hline \multirow{2}{*}{ Class } & \multicolumn{2}{|c|}{ BT-MZ } & \multicolumn{2}{|c|}{ LU-MZ } & \multicolumn{2}{|c|}{ SP-MZ } & \multirow{2}{*}{$\begin{array}{l}\text { Aggregated } \\
\text { Grid Size }\end{array}$} & \multirow{2}{*}{$\begin{array}{c}\text { Memory } \\
\text { Requirements }\end{array}$} \\
\hline & no. zones & no. iters & no. zones & no. iters & no. zones & no. iters & & \\
\hline B & $8 \times 8$ & 200 & $4 \times 4$ & 250 & $8 \times 8$ & 400 & $304 \times 208 \times 17$ & $\approx 200 \mathrm{MB}$ \\
\hline C & $16 \times 16$ & 200 & $4 \times 4$ & 250 & $16 \times 16$ & 400 & $480 \times 320 \times 28$ & $\approx 0.8 \mathrm{~GB}$ \\
\hline $\mathrm{D}$ & $32 \times 32$ & 250 & $4 \times 4$ & 300 & $32 \times 32$ & 500 & $1632 \times 1216 \times 34$ & $\approx 12.8 \mathrm{~GB}$ \\
\hline
\end{tabular}

TABLE 1

Characterization of NPB-MZ input classes: number of zones and number of time-step iterations (per each benchmark), 3D-mesh size and memory requirements.

The size of a zone is determined by the product of the sizes of its $\mathrm{x}, \mathrm{y}$ and $\mathrm{z}$ dimensions ( $\mathrm{z}$ is common to all zones). NPB-MZ benchmarks exhibit significant differences in both the number and the size of the zones they deal with. LUMZ benchmark creates exactly 16 zones (independently of the input class) of equal size. In both SP-MZ and BT-MZ benchmarks, the bigger the input class, the larger the number of zones; however, while SP-MZ manages zones of equal size, BT-MZ deals with zones that show of a wide range of sizes. These differences will influence on the performance effect of our parallelization on each benchmark. 


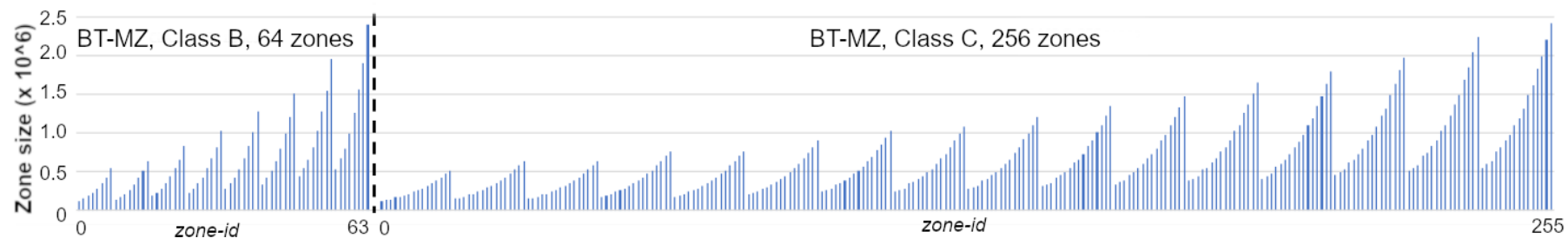

Fig. 2. Size of each zone in BT-MZ benchmark (input classes B and C). Zones are sorted by its zone-id from 0 up to the number of zones minus one. Zone sizes are different in factors up to a 19x factor, meaning that the biggest zone is 19 times larger than the smallest zone.

Fig. 2 shows the size of each zone in BT-MZ benchmark for input classes B and C; zones are sorted according to their zone-id. The saw-based pattern is repeated several times with an increasing maximum size difference within each instance of the pattern. Also, while in class B the pattern is composed of 8 zones, in class $C$ the pattern is formed with 16 zones. Class D follows the same pattern with 1024 zones organized in 32 zones per pattern instance. Through this work we will take into account this behaviour to achieve a balanced workload distribution for BT-MZ benchmark.

\subsection{Computation/Communication phases}

The execution of NPB-MZ benchmarks follows a common pattern. First, data structures (e.g.: matrices representing the zones and temporary matrices that hold intermediate results) are allocated and initialized. Next, a time-step loop that contains both the computational and the communication phases of the benchmark solves a numerical method; the number of loop iterations depends on the benchmark and input class (TABLE 1). Finally, the benchmark verifies the outcome of the execution for correctness checking.

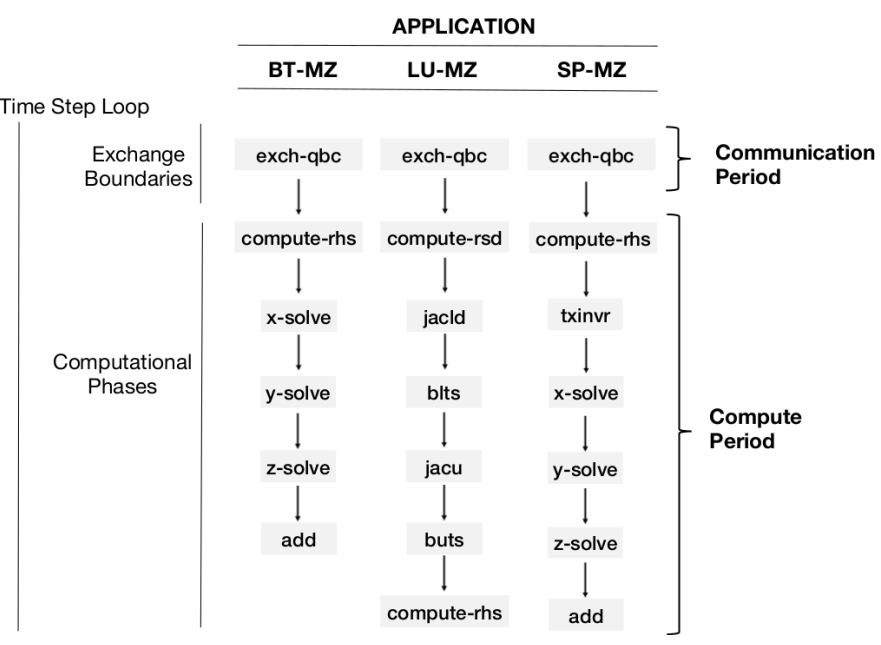

Fig. 3. Time-step loop of NPB-MZ benchmarks: sequence of computation/communication phases per benchmark.

Fig. 3 shows the sequence of computation and communication phases per each application. For BT-MZ and SP-MZ, the computational phases are very similar because the numerical method implemented in both applications is alike. All applications alternate a sequence of several computational phases with one communication phase (e.g.: exch-qbc) where boundary values between the zones are interchanged and recomputed according to the adjacency of the zones (Fig. 1). Here after, we define the Compute Period as the set of computational phases and the Communication Period as the boundary-exchange computation phase.

The serial implementation of the Compute Period consists in a sequence of iterative structures, with as many structures as the number of computational phases. Each iterative structure defines a loop that processes one zone per iteration, and executes a fixed computational phase over the zone. For a phase and a zone, the computations are implemented with several loop nests that traverse and compute multidimensional matrices associated to the processed zone. The sequence of phases is computed sequentially.

The implementation of the Communication Period requires processing the zones sequentially, from the zone with zone$i d=0$ to the zone with the largest zone-id. For each zone, its adjacent 2D surfaces must be copied into temporary matrices. Then the solution for these boundary values is computed and copied back to their original storage. Uppermost side of Algorithm 2 depicts this pseudo-code.

\subsection{Sources of parallelism}

The available parallelism in NPB-MZ benchmarks can be classified as inter-zone and intra-zone parallelism. While the Compute Period exposes both kinds of parallelism, the Communication Period exposes only intra-zone parallelism. The following subsections describe the origin and the nature of each type of parallelism in the NPB-MZ suite.

\subsubsection{Compute period: Inter-zone Parallelism}

The left side in Algorithm 1-a shows the skeleton of the time-step loop of the NPB-MZ applications. Each computational phase of the benchmarks is implemented with a loop (zone-phase loop) that traverses the zones and processes them. As processing a zone is independent of processing the remaining zones, the exploitation of inter-zone parallelism implies the parallelization of these loops.

The right side of Algorithm 1-a shows the OpenMP implementation for such parallel strategy. A parallel region is defined per each zone-phase loop. The parallel region is executed with as many threads as GPUs are used for the multi-GPU execution. Under this execution model, there is one OpenMP thread that controls both the computation and the communication associated to one GPU. The body of this parallel region is composed of a while loop statement where at each iteration the runtime checks for work availability to be offloaded to a GPU. According to the applied scheduling, the iterations of the zone-phase loop are distributed to threads. Each OpenMP thread translates the assigned iterations to zones. Zones assigned to a particular thread 
Algorithm 1 a) Inter-Zone Parallelism: The left side shows the code skeleton for the time-step loop. Its body is composed of as many loops (zone-phase loops) as the number of phases. Each zone-phase loop traverses the zones and applies a phase over a zone on each iteration. The right side shows the OpenMP implementation that distributes the iterations of the zone-phase loops (e.g.: the zones) to the GPUs. An OpenMP parallel region is defined and one thread controls one CPU, executing the body of the parallel region. The while loop invokes the runtime system to acquire work.

b) Intra-Zone Parallelism: Each phase is coded as a subroutine where iterative structures implement a computation over the multi-dimensional matrices that represent one zone. Each iterative structure is converted into a CUDA kernel (Kernel Definition), and substituted by a kernel CUDA call (Kernel Invocation). Each kernel operates only over a single zone.

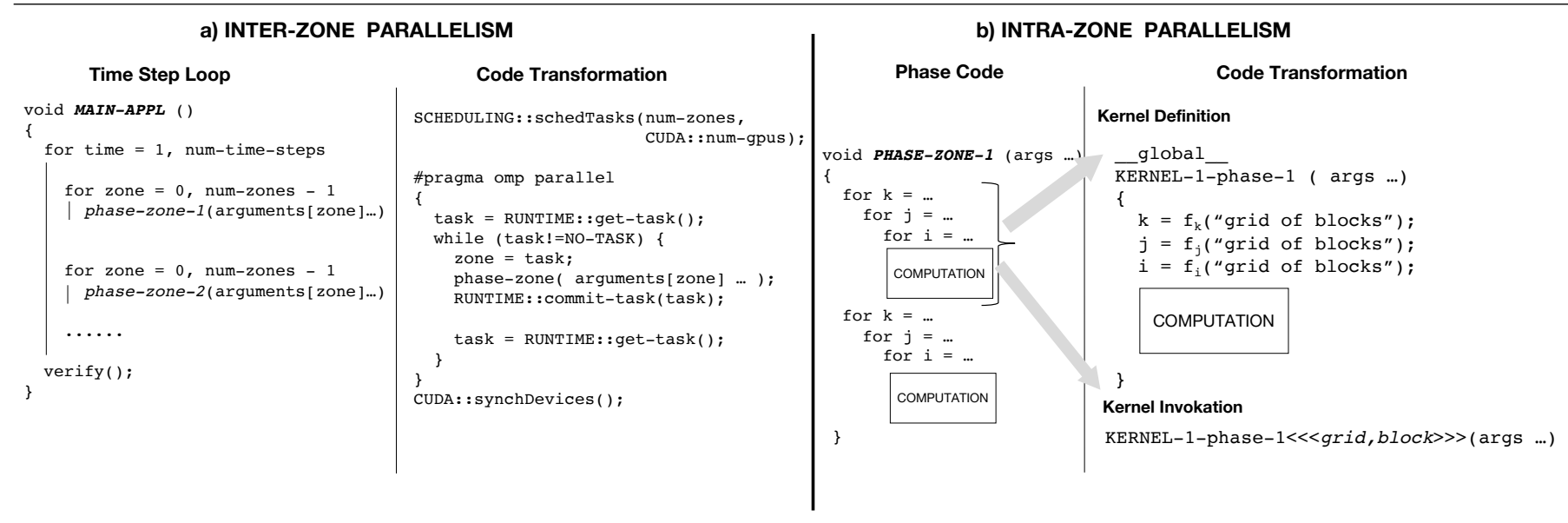

will be executed on the GPU the thread is responsible of. The runtime invocations to get-task and commit-task modify internal variables of the runtime system to monitor whether a zone has already been processed or not.

\subsubsection{Compute period: Intra-zone Parallelism}

Left side of Algorithm 1-b shows that each iteration of the zone-phase loops calls a subroutine with several loop nests that implement the computation for one zone and a computational phase. The intra-zone parallelism corresponds to the exploitation of the parallelism exposed by these loop nests.

The loop body of each loop nest is converted to a CUDA kernel and the iteration space is translated, at kernel invocation time, to a CUDA < grid, block> definition for CUDA threads organization. This approach is based on previous work [7] where single-GPU implementation is developed for NPB and NPB-MZ benchmark suites.

Algorithm 1-b depicts the transformation from a loop nest to a CUDA kernel definition and invocation. Per each nest, its transformation into a kernel requires the definition of functions that implement the association of CUDA threads to actual elements in the data structures (e.g.: matrices) that represent the zones. These functions are kernel specific. In general, each computational phase includes several loop nests, therefore each phase is composed of as many CUDA kernels as parallelized nests. All kernel invocations target the default CUDA stream and, whenever possible, shared memory has been used.

\subsubsection{Communication period: Intra-zone Parallelism}

The original algorithm for the exchange of boundary values is applied over all zones sequentially (see uppermost side in Algorithm 2). As this computation forces processing the zones one after another, it is not possible to exploit inter-zone parallelism. However, some parallelism is exposed in the computations within a zone. This intra-zone parallelism can be exploited through the definition of kernels that implement the border computation. For single-GPU execution this is similar to the phase parallelization described in section 2.4.2 (middle part of Algorithm 2). Notice that each loop iteration processes one zone and updates the boundaries with only two of its adjacent zones (east and north adjacent zones). The boundaries with the remaining adjacent zones (west and south) will be updated in the loop iterations where west and south zones are processed.

Porting the boundary-exchange computations to multiGPU requires the introduction of data-transfer statements. The border computation for one zone is performed in a particular GPU and it is necessary to check if all adjacent zones reside in that GPU. If not, the missing zones must be transferred to the GPU. As the border computation updates both the zone and its adjacent zones, replicated zones must be copied back to their original location. Lowermost side of Algorithm 2 shows the pseudo code for the multi-GPU boundary-exchange algorithm. Note the use of communication statements (CUDA::copy-zone function) between the kernels. These statements perform memory copy operations between the devices that store adjacent zones. It is worth mentioning that, according to distribution of the zones to the GPUs, copy statements might be unnecessary. They are necessary only when adjacent zones are mapped to different GPUs. For simplicity, we have excluded the code and data structures associated to the zone-placement management, and assumed the zone placement be managed within the call to the copy statement. The impact of this communication is a key aspect of study as well as understanding the relation between work-distribution schemes (zone-to-GPU mapping) and their effect in the execution of the boundaryvalues exchange algorithm. Next section describes the workdistribution schemes proposed in this study. 


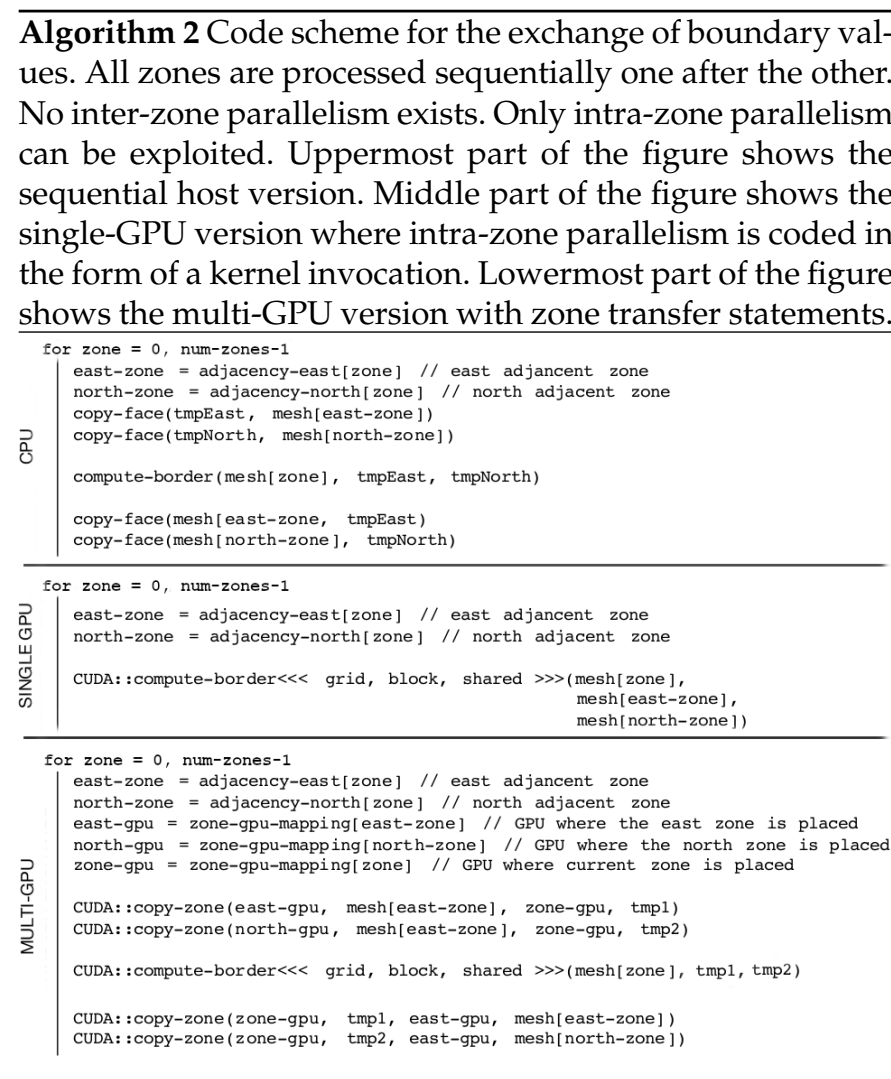

\section{Computation/Communication Trade-OfF AND WORK SCHEDULING}

This section describes the trade-off between the distribution of zones across the GPUs and the data transfers needed to perform the boundary-values computation. As described in Fig. 2, zones are non uniformly sized resulting in potential work unbalance. Besides, depending on the zone-to-GPU mapping, the exchange of boundary values generates different communication patterns. Note that this mapping will determine which zones must be transferred between GPUs when executing the boundary computations (see lowermost part of Algorithm 2). Therefore, the zone distribution affects simultaneously the work unbalance and the data transfers to happen during the boundary-values computation.

To address both issues and study their relationship, we study three work-distribution schemes. 1) a Static scheduling that minimizes the scheduling overhead but does not take into account neither the size of the zones nor the zone adjacency. 2) a Dynamic scheduling that mainly addresses work unbalance, but does not consider zone communications because it is not guided by any adjacency criteria. As this scheduler has a parameter (the chunk size), we explore its design space for work granularity. In addition, this scheduler memorizes the work distribution across its instances, which makes it a variant of dynamic self schedulers [8]. 3) we propose a variant of the Guided scheduling that simultaneously tries to balance the execution among GPUs and to minimize both the scheduling and communication overheads. This loop scheduler corresponds to a novel adaptation of feedback-based dynamic loop schedulers [8].

\subsection{Static scheduling}

This scheduling assigns zones evenly among the GPUs: the total number of zones is divided by the number of GPUs. Then, the same number of consecutive zones (according to the iteration space defined in the zone-phase loops, see Algorithm 1-a) is assigned to each GPU. Note that given the zone adjacency described in section 2.4.3 (see also Fig. 1), this scheduling assigns a group of contiguous zones in the $x$-dimension to each GPU. Then, most communications are due to y-dimension adjacencies; only first and last zones in the group require $\mathrm{x}$-dimension adjacencies from other GPUs.

In Algorithm 1-a, the call to SCHEDULING::schedule-tasks pre-assigns the group of zones per each GPU. Then, the while statement iterates as many times as zones are mapped to a GPU (e.g.; the number of zones in the group). The RUNTIME::get-task and RUNTIME::commit-task calls manage the mapping between iterations and actual zones.

If this scheduling leads to a workload unbalance, the scheduling will not try to balance the execution time among the GPUs. In contrast, this scheduling generates data transfers just for one dimension which might minimize the total number of data transfers during the Communication Period. Notice that this scheduling keeps invariant the zone-to-GPU mapping across the instances of the Compute Period. Therefore, the number of zone transfers for the Communication Period will be also constant and invariant.

\subsection{Dynamic scheduling}

This scheduling assigns zones to GPUs dynamically at runtime. It divides the iteration space of the zone-phase loops into chunks of chunk consecutive zones; chunk is a predefined parameter of the scheduler. A chunk of zones is assigned to each GPU. As soon as a GPU finishes processing its chunk, the scheduling assigns a new chunk to the GPU.

Threads acquire portions of the iteration space of the zone-phase loops. The call to SCHEDULING::schedule-tasks in Algorithm 1-a does not pre-assign any zone to any GPU. Per each iteration of the while statement, a chunk of iterations are assigned to a GPU. In general, this scheduling will tend to balance the execution time if zone sizes are different, but will map the zones across the GPUs so that adjacency is not respected neither in the $x$-dimension nor in the $y$-dimension. Therefore, this scheduling will generate data transfers for adjacent zones in the $y$ and $x$ dimensions, potentially enlarging the total execution time for the Communication Period.

The chunk parameter can smooth the effect on the data transfers. The scheduling will assign chunk adjacent zones in the $\mathrm{x}$-dimension as the minimal work unit to be distributed. Thus, the bigger the chunk, the less communications but, potentially, the larger the work unbalance.

Notice that this scheduling may change the zone-to-GPU mapping on each of the instances of the Compute Period. Thus, the number of zone transfers will be also variant and dependant on this mapping.

In order to balance the Compute Period and simultaneously keep constant and low the number of zone transfers, we have implemented a memorizing variant of the feedback dynamic loop scheduler [8], a state-of-the-art dynamic loop scheduling. After a few instances of the Compute Period, the scheduling memorizes the zone-to-GPU mapping and 
keeps it invariant for the remaining instances. The aim of this variant is avoiding zone migrations between GPUs. So, this characteristic makes it an affinity-oriented scheduler. Its dynamic behaviour and chunk parameter also provides the ability of balancing the work distribution across the GPUs. If we assume the mesh zones as parallel tasks, the loop scheduling can be also seen as a task scheduler where the chunk parameter controls the granularity of the tasks.

\subsection{Guided scheduling}

Like the Static scheduling, this scheduling distributes the zones in as many chunks of consecutive zones as the number of GPUs. However, the chunks may not contain the same number of zones. The iteration space of the zone-phase loops is cut into chunks of consecutive iterations. The points in the iteration space where the chunks start/end are determined by the total amount of work in each chunk. We describe two variants of this scheduling.

On one hand, Guided-Sizes estimates the workload in a chunk by accumulating the sizes of the zones in the chunk. Like the Static scheduling, this scheduling assigns to each GPU zones that are adjacent in the $\mathrm{x}$ dimension (except for the first and the last zones in a chunk). However, the amount of work is balanced according to the sum of the zone sizes.

On the other hand, Guided-Runtime estimates the workload in a chunk by accumulating the execution time of its zones. At runtime, the scheduler collects the execution time of each zone excluding any communication related to the zone allocation and boundary-values computation. Then, the scheduling uses this information to balance the execution time among GPUs. Like the Guided-Sizes scheduling, communications appear only across the $y$-dimension, not the $x$-dimension.

Algorithm 3 shows the algorithm for the Guided schedulers. The algorithm takes an input vector that represents the workload of each zone and another input vector that represents the workload performed by each GPU in the previous instance of the Compute Period. These vectors describe the work in terms of size (Guided-Sizes) or time (GuidedRuntime). The total workload is computed (variable TotalWork) by accumulating the workload of each GPU, and then the ideal balanced workload per GPU (variable WorkPerGpu) is obtained dividing the total work by the number of GPUs (see Algorithm 3 from line 1 to 3). Also, the algorithm takes as input/output two vectors that represent the current chunk limits (first and last zone in the chunk of consecutive zones). Then, the algorithm (loop from line 4 to 31) updates the current chunk limits to improve work balance. Given a GPU, if the work done is less than the estimated WorkPerGpu (line 8) the algorithm tries to move up the index for the last zone in the chunk of zones assigned to the GPU (lines 9-17). This is implemented in the while loop (line 9) that increments variable Last as long as the assigned workload to the GPU gets closer to WorkPerGpu. If the workload is greater than WorkPerGpu, then the algorithm tries to move down the index of the last zone in the chunk of zones assigned to the GPU (lines 19-27). Similarly, the while loop (line 19) decrements variable Last as long as the assigned workload to the GPU gets closer to WorkPerGpu.
Algorithm 3 Algorithm for Guided schedulers. Input: the work done per gpu (WorkDonePerGpu), the work in each zone (WorkPerZone). Input/Output: indexes for first and last zone to be processed by each GPU (IndexFirstZone, IndexLastZone). The indexes define chunks of consecutive zones along the global numbering of zones.

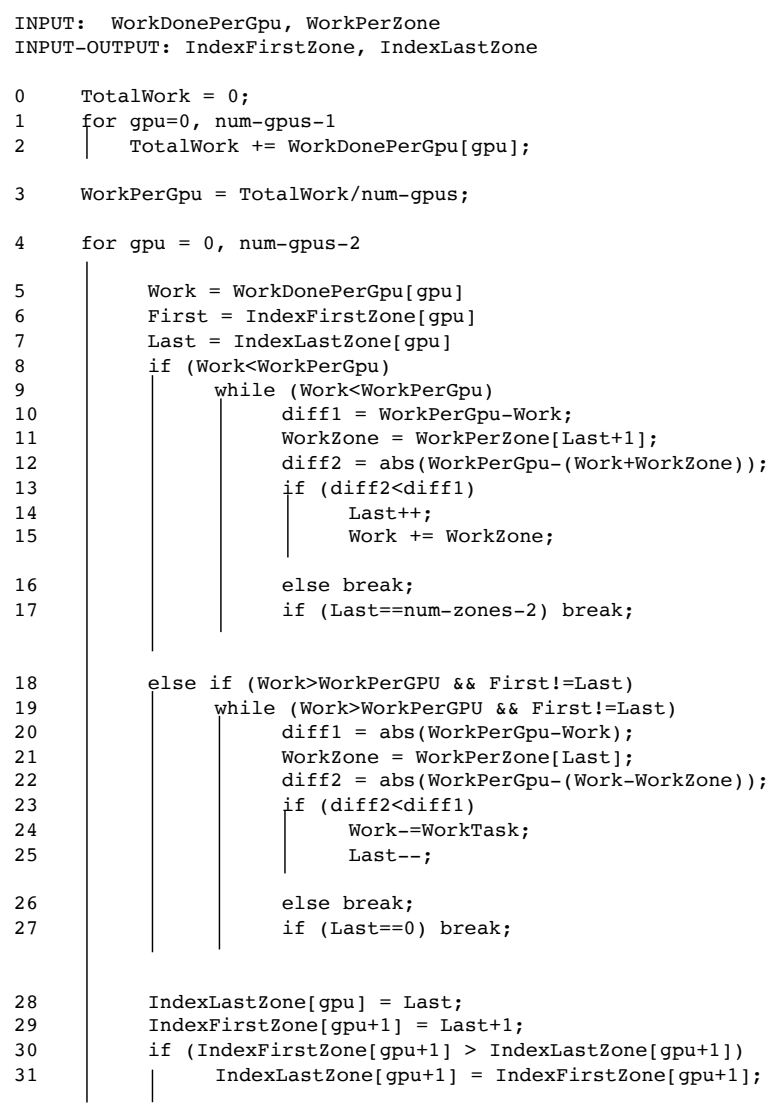

\subsubsection{Guided scheduling versus optimal scheduling}

The guided scheduler maps zones to GPUs at run-time using profile information collected on the previous iteration of the time-step loop. In order to be affordable at runtime, the guided scheduler is designed for simplicity. In this subsection we wonder about the feasibility of finding the optimal scheduling for our benchmarks, which will be used, for comparison purposes, in the evaluation section.

We have modeled the problem of finding the optimal scheduling as an 0-1 integer-linear optimization problem [9]. Given the array of zones, each zone must be mapped to a GPU. The cost of each zone is twofold: firstly, a computation cost that is proportional only to the zone size; secondly, a communication cost that arises if the neighbor zones (Fig. 1) are not mapped to the same GPU. The load of each GPU is the sum of the costs of all the zones mapped to the GPU. Consequently, finding the optimal scheduling is equivalent to finding a zone-to-GPU mapping that minimizes the load of the most loaded GPU: this is the objective function of the optimization problem. As the zone sizes of NPB-MZ benchmarks are known at compile time, we are able to solve the optimization problem off-line and provide the resulting scheduling to the benchmark at run-time.

To solve the optimization problem, we use IBM ILOG 
CPLEX solver [10] with default parameters on a Linux server running on a Intel(R) Xeon(R) CPU E3-1220 v3 @ 3.10GHz processor. Obtaining the optimal scheduling for BT-MZ benchmark takes about 2 minutes (B class) and 2 hours (C class). Unfortunately, the time needed to obtain the solution for class D is too large; however, in the evaluation section we will show how to extrapolate to class $D$ the performance trends observed in classed $\mathrm{B}$ and $\mathrm{C}$.

Fig. 4 displays the zone-to-GPU mappings obtained by both the guided (left) and the optimal (right) schedulers for classes B and C on BT-MZ benchmark. Equally colored zones are mapped to the same GPU. Note that, as shown in Fig. 2, zones in BT-MZ are not equally sized; for instance, bottom-right zone is about 20x bigger than top-left zone.
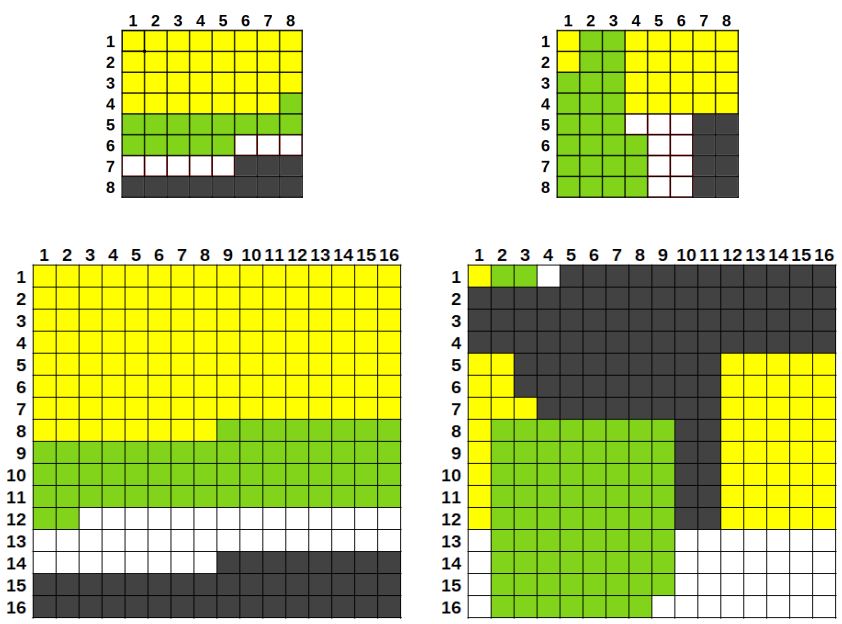

Fig. 4. Zone-to-GPU mappings achieved in BT-MZ by guided (left column) and optimal(right column) schedulers, input class B (top row) and C (bottom row). Equally colored zones are mapped to the same GPU.

The diagrams show that the guided scheduler is restricted to map zones with consecutive zone-id's to each GPU. In contrast, the optimal scheduler can map any zone to any GPU. In both cases, the cuts they achieve try to maximize adjacency while keeping the work balanced in the Compute Period. Guided scheduler cuts the mesh exclusively along y-dimension. The optimal scheduler is able to cut the mesh in both the $y$ and $x$ dimensions.

On the results section we will discuss the performance lost due to using the guided scheduler instead of using the optimal scheduler on BT-MZ benchmark.

\section{Results}

This section presents an in-depth analysis performance of our multi-GPU parallelization of the NPB-MZ benchmarks.

\subsection{Evaluation environment}

Our parallelization has been evaluated in a system composed of 2 x IBM Power9 8335-GTH @ 2.4GHz $(3.0 \mathrm{GHz}$ on turbo, 20 cores and 4 threads/core, total 160 threads per node) with 512GB of main memory distributed in 16x 32GB DIMMS @ 2666 MHz and 4 x GPU NVIDIA V100 (Volta) with 16GB HBM2. The software environment is Red Hat Enterprise Linux Server 7.5 alternative, CUDA 10.1 compiler, CUDA 418.39 driver and GCC 4.8.5.

\subsection{Performance Analysis}

We characterize the performance of our parallelization of the NPB-MZ applications in terms of their work-unbalance degree and the overall number of data transfers between the GPUs. We analyze separately the two periods of execution of the applications (Compute period and Communication period, Fig. 3) because they show very different patterns in terms of computation and communication. Unless otherwise noted, all the experiments have been realized with 4 GPUs. We have used CUDA events to trace the two periods, and all data transfers from/to devices and. host are synchronous.

\subsubsection{BT-MZ benchmark}

In BT-MZ application, as noted in Fig. 2, the zones show a wide range of sizes and, the greater the input class, the larger the number of zones. We must consider this behaviour to understand the performance results of BT-MZ.

\section{Compute Period}

Fig. 5 shows, for each input class and scheduler, the duration of the computation and communication bursts performed by each GPU on one instance of the Compute Period. This figure depicts work-balance differences between the schedulers. To quantify the work unbalance (w.u.), we define w.u. metric as the ratio between the execution time of the GPU that takes the maximum time and the execution time of the GPU that takes the minimum (w.u. $=1$ is a perfect balance).

Using the Static scheduler, the work unbalance is evident in all input classes: w.u. ranges from 2.26 to 3.28 . It is due to the combination of two factors: zone sizes are not uniform and each GPU processes the same number of zones.

The Dynamic scheduler tries to balance the workload among the GPUs by deciding at run-time which GPU must process each zone. Our first evaluations showed that, although the Dynamic scheduler succeeded at balancing the workload, it also significantly degraded the performance. Fig. 6 shows the workload distribution achieved by the Dynamic scheduler with chunk 1 while executing one instance of the class B Compute Period. While the figures related to the Static scheduler show few Communication bursts, the Dynamic scheduler produces a significant amount of Communication bursts that enlarges the execution time with respect the Static scheduler ( $\sim 20 \mathrm{~ms}$ versus $\sim 12 \mathrm{~ms}$ respectively). These communications correspond to zone migrations from the GPU where a zone has been assigned for computation in the previous Compute Period to the GPU where the zone has been assigned in the current Compute Period. To address this issue, the Dynamic scheduler applies "memorization", that is, it keeps constant the zone assignment resulting after a few instances of the Compute Period.

Once the Dynamic scheduling keeps constant the zoneto-GPU mapping, performance degradation disappears and workload balance remains. Fig. 5 shows the performance obtained by the Dynamic scheduler for different values of the chunk parameter $(1,2,4,8$ and 16 zones). For class B and chunk 1, the execution time of the Compute Period is $11.0 \mathrm{~ms}$; for class C is $33 \mathrm{~ms}$ and for class D $490 \mathrm{~ms}$. Work unbalance is reduced compared to Static scheduling; it ranges from 1.10 to 1.62. We observe that the smaller the chunk, the longer the communications in the Compute Period. 


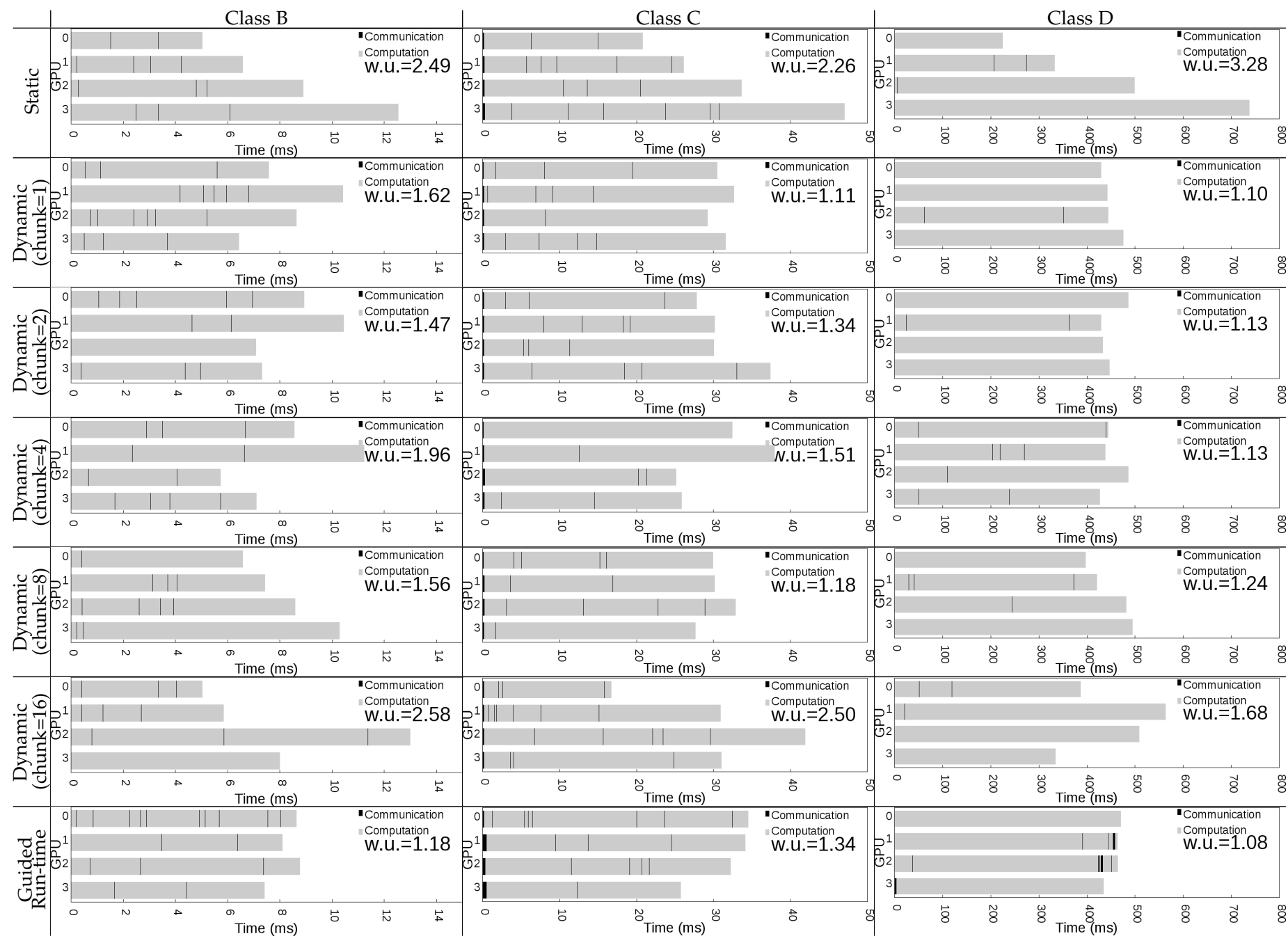

Fig. 5. Influence of the scheduler on the work balance of the Compute Period on BT-MZ. For each input class and scheduler, a histogram shows the duration of computation and communication bursts at each GPU. Work unbalance (w.u. $\geq 1$, the smaller the better) is shown for each histogram.

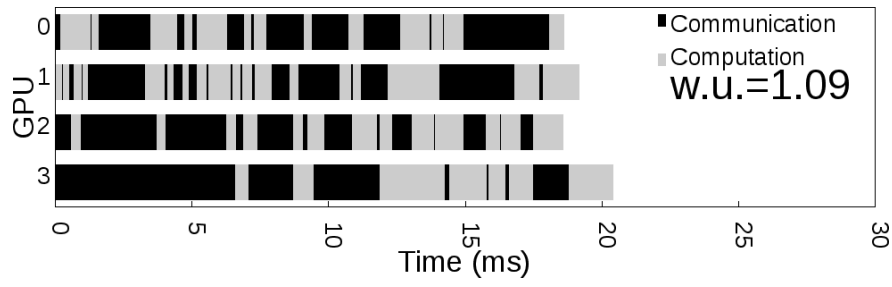

Fig. 6. Work balance induced by the Dynamic scheduler without memorization in BT-MZ (class B, chunk 1). Computation and communication are interleaved. Darker zones indicate communication bursts due to zone transfers between the GPUs.

In contrast, we have observed that the smaller the chunk the better the work balance, as it should be expected. In particular for class D, the Dynamic scheduler succeeds in practically balancing the execution of the Compute Period no matter the chunk value (this class generates 1024 zones). For smaller classes (B and C), only the variants with chunk 1, 2, 4 and 8 expose an improvement of the work unbalance. But this improvement is conditioned by the number of zones and the way the application assigns the zone sizes. For class B, only 64 zones are available, insufficient even when executing with chunk 1 . For class C, 256 zones are available so, with smaller chunks, better work-balance is observed than when executing with chunk 16.

The Guided schedulers behave like the Dynamic scheduler but with a better work balance. Guided schedulers balance the work across the GPUs. For the Guided-Runtime scheduler, w.u. is $1.18,1.34$ and 1.08 for classes B, C and $\mathrm{D}$ respectively. Also, communication bursts are seldom because the portions of work per each GPU remain constant and well balanced. This happens after several instances of the Compute Period, where the scheduler reachs a balanced distribution. We have checked that Guided-Sizes scheduler presents a very similar behavior, therefore it is not shown.

In conclusion, the Static scheduler does not succeed on balancing workload. In contrast, Dynamic and Guided schedulers generate well balanced work distributions, but with different degrees of communication along the Compute Period. Dynamic schedulers without memorization often generate zone transfers; this is solved by the memorization. Guided schedulers capture the appropriate work distribution with no need of zone transfers along the Compute Period. In all cases, we have observed that the overhead due to the 
execution of the loop scheduler is negligible in comparison with the work granularity exposed by the scheduled loops. This is significant, specially for the Dynamic with memorization and the Guided schedulers.

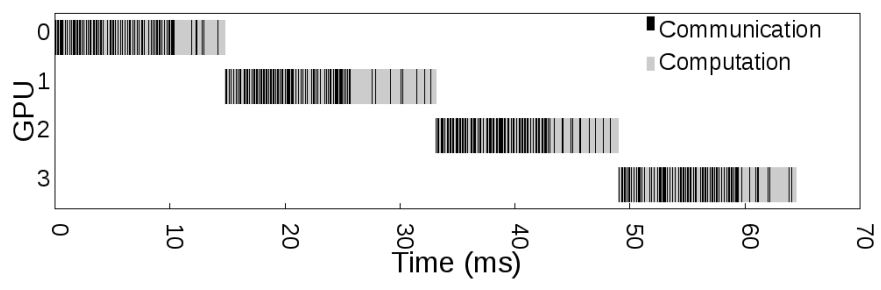

Fig. 7. Work distribution among the GPUs during the Communication Period (static scheduler). Notice the serialization of the computation: zones are processed one after the other in the GPU where each zone is placed. Processing of one zone might imply a zone transfer of their neighboring zones (darker bursts in the figure).

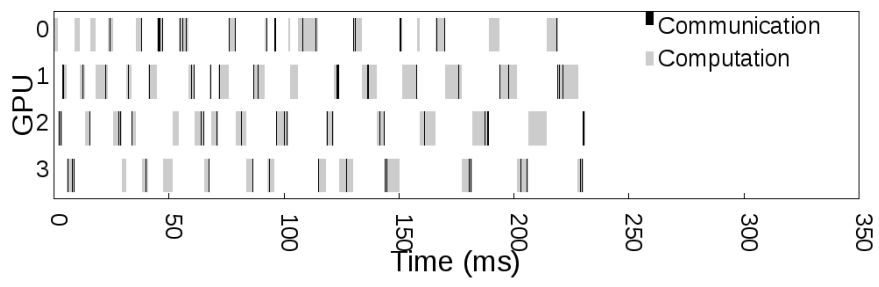

Fig. 8. Work distribution among the GPUs during the Communication Period (dynamic scheduler, chunk 16). The sequential zone processing and the zone-to-GPU mapping generated by the dynamic scheduler spreads computation and communication bursts across the GPUs.

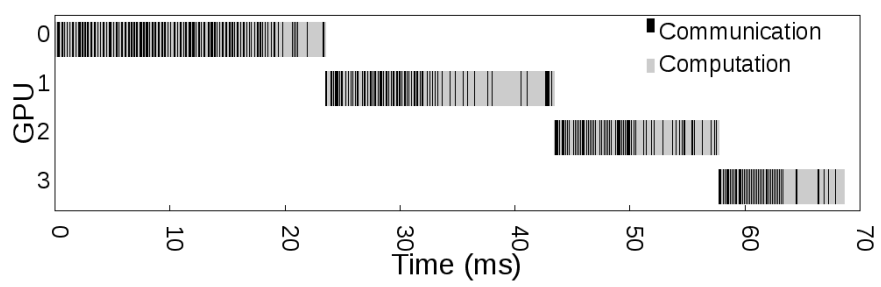

Fig. 9. Work distribution among the GPUs during the Communication Period (guided scheduler). Behaves like the Static scheduler, but the Guided scheduler is able to balance the Compute Period.

\section{Communication Period}

The computation corresponding to boundary values has no inter-zone parallelism: zones are processed sequentially. The main aspect to consider is the amount of zone transfers among GPUs, that depends on the zone-to-GPU mapping decided by the scheduler in the the Compute Period.

Fig. 7 depicts the behaviour of the Communication Period after a Compute Period statically scheduled. As zones from zone- $i d=0$ to $z o n e-i d=n u m z o n e s / 4-1$ have been assigned to GPU 0, GPU 0 starts processing these zones and generates communication bursts mainly caused by the zone adjacency in the y-dimension. After GPU-0 has processed its assigned zones, GPU 1 continues the execution exposing the same pattern. In general, a sequential pipeline is generated for all GPUs, and the static scheduler causes the Communication Period to be completed in $65 \mathrm{~ms}$.

In contrast, Fig. 8 shows the behaviour of the Communication Period after a Compute period dynamically scheduled with chunk 16. Data transfers occur almost for every processed zone as the adjacent zones for both $\mathrm{x}$ - and $\mathrm{y}$ dimensions are resident in a different GPUs than the one processing the current zone. The Dynamic scheduler in the Compute Period makes the Communication Period to execute in about $230 \mathrm{~ms}$ ( $3.5 \mathrm{x}$ slower than the Static case).

Fig. 10 shows the total number of zone transfers during the Compute and Communication Periods. The Static scheduler causes much less zone transfers than the Dynamic schedulers (in fact the differences are of an order of magnitude). Note the expected behaviour related to the chunk size: the bigger the chunk, the fewer communications are generated.

Finally, the Guided schedulers behave like the Static scheduler. These schedulers assign consecutive zones to GPUs. This makes contiguous zones to be assigned to the same GPU, so minimizing zone transfers across the $x$ dimension. In general, these schedulers place the zones so that the Compute Period gets well balanced and the Communication Period is not affected by a sub-optimal number of zone transfers. Fig. 9 shows the behaviour of the Communication Period after a Compute Period scheduled by a Guided scheduler. Its execution time $(69 \mathrm{~ms})$ is similar to the one obtained with the Static scheduler. Fig. 10 accounts the zone transfers for this scheduler type, having a total number of transfers similar to the Static scheduler.

\section{Compute Period vs Communication Period}

The left side of Fig. 11 shows the execution times for the Compute Period and Communication Period as well as their relative contribution to the overall execution time of one iteration of the time-step loop of BT-MZ. The figure explores all input classes, schedulers and uni-GPU, 2-GPU and 4GPU executions. Moreover, the right side of the figure shows the speedup at each period achieved by the 2-GPU and 4GPU execution with respect to the uni-GPU execution.

For class B and Static scheduler, the Compute Period takes $35.20 \mathrm{~ms}, 25.30 \mathrm{~ms}$ and $16.00 \mathrm{~ms}$ using 1,2 and 4 GPUs respectively. This accounts for $83 \%, 80 \%$ and $73 \%$ as the relative contribution. So, clearly, the Communication Period is increasing its relative contribution not only because the execution time of the Compute Period shortens, but also because the Communication Period takes longer. The later processes sequentially the zones, so its performance degradation is due to the communications between the GPUs. While the Compute Period speeds up by 1.39x and 2.20x (2 and 4 GPUs resp.) the Communication Period degrades to $0.82 \mathrm{x}$ and $0.67 \mathrm{x}$ resp. The combination of both behaviors determines the overall speedup, studied in next section.

Dynamic schedulers show a similar trend but with better speedups in the Compute Period and worse slowdowns in the Communication Period. Speedups range from 1.74x and $2.71 \mathrm{x}(2,4$ GPUs with chunk 1$)$ to $1.57 \mathrm{x}$ and $2.07 \mathrm{x}(2,4$ GPUs, chunk 16). Performance in the Communication Period degrades to $0.48 \mathrm{x}$ and $0.36 \mathrm{x}(2,4$ GPUs, chunk 1$)$, and to $0.72 \mathrm{x}$ and $0.67 x$ (2, 4 GPUs, chunk 16). Clearly, while the Dynamic scheduler outperforms the Static scheduler on the Compute Period, it is outperformed on the Communication Period. 

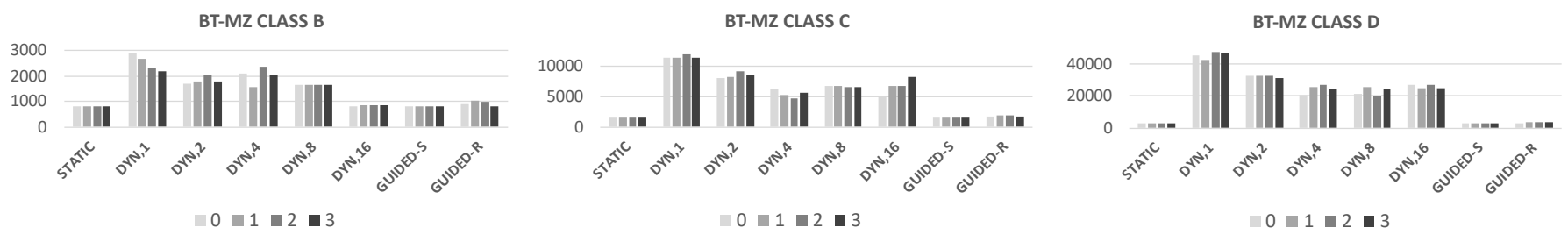

Fig. 10. Number of zone transfers per GPU in both Compute and Communication Periods for all schedulers. Observe that Static and Guided schedulers have by far less transfers than the Dynamic schedulers. Zone placement determines the number of zone transfers for the Communication Period. While dynamic schedulers scatter the zones over the GPUs, Static and Guided schedulers gather consecutive zones in the same GPU.
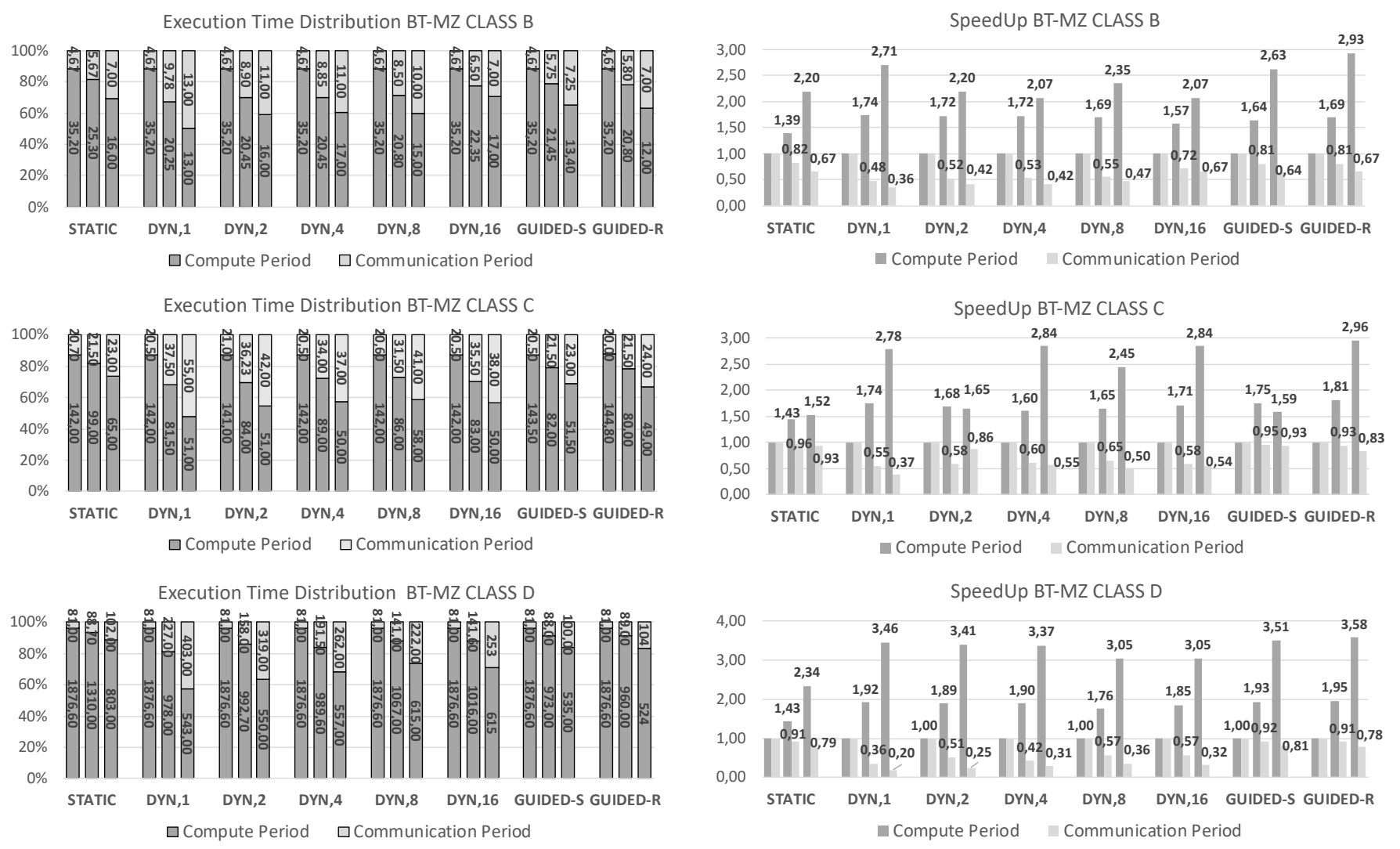

Fig. 11. Left side: Execution time distribution into Compute and Communication Periods for all schedulers using 1, 2, and 4 GPUs. Figures inside the bars stand for the duration (ms) of the period. Right side: Speedup observed at each period with respect to uni-GPU execution.

Finally, Guided schedulers improve similarly the Compute Period (speedup ranges between $1.64 \mathrm{x}$ and $2.93 \mathrm{x}$ with 2 and 4 GPUs), but the performance degradation in the Communication Period is similar to the Static case, and much smaller than the Dynamic cases. Notice that when comparing the two Guided variants we have not observed a significant difference in performance, although the variant Runtime is always faster (less than $10 \%$ faster).

For classes $C$ and $D$, the trends are very similar to class B. The fastest schedulers are the Guided variants, both performing again in a very similar way. For these larger classes, the Dynamic variants suffer from even more slowdown on their Communication Period as the number of zones is increased and the zones are larger: in class D, Communication Period performance degrades to $0.36 \mathrm{x}$ and $0.2 x$ ( 2 and 4 GPUs with chunk 1$)$, and to $0.57 x$ and $0.32 x(2,4$ GPUs with chunk 16). Next section addresses the combined effect of improving the Compute Period while degrading the Communication Period.

\section{Overall Performance}

Fig. 12 shows the overall speedup achieved by the 2-GPU and 4-GPU executions with respect to the uni-GPU execution. The figure explores all the schedulers and input classes.

For class B, the fastest schedulers are the Guided variants that outperform uni-GPU execution by $1.64 \mathrm{x}$ and $2.39 \mathrm{x}$ (2 and 4 GPUs, Guided Sizes) and 1.77x and 2.51x (2 and 4 GPUs, Guided Runtime). Dynamic schedulers are not able to achieve more than $1.5 \mathrm{x}$ with 2 GPUs nor more than $2.03 x$ with 4 GPUs. The reason for this behaviour has been explained in the previous section, where we have observed the essential slowdown in the Communication Period for these schedulers. The Static scheduling performs at the same level than the Dynamic variants because, although not suffering 


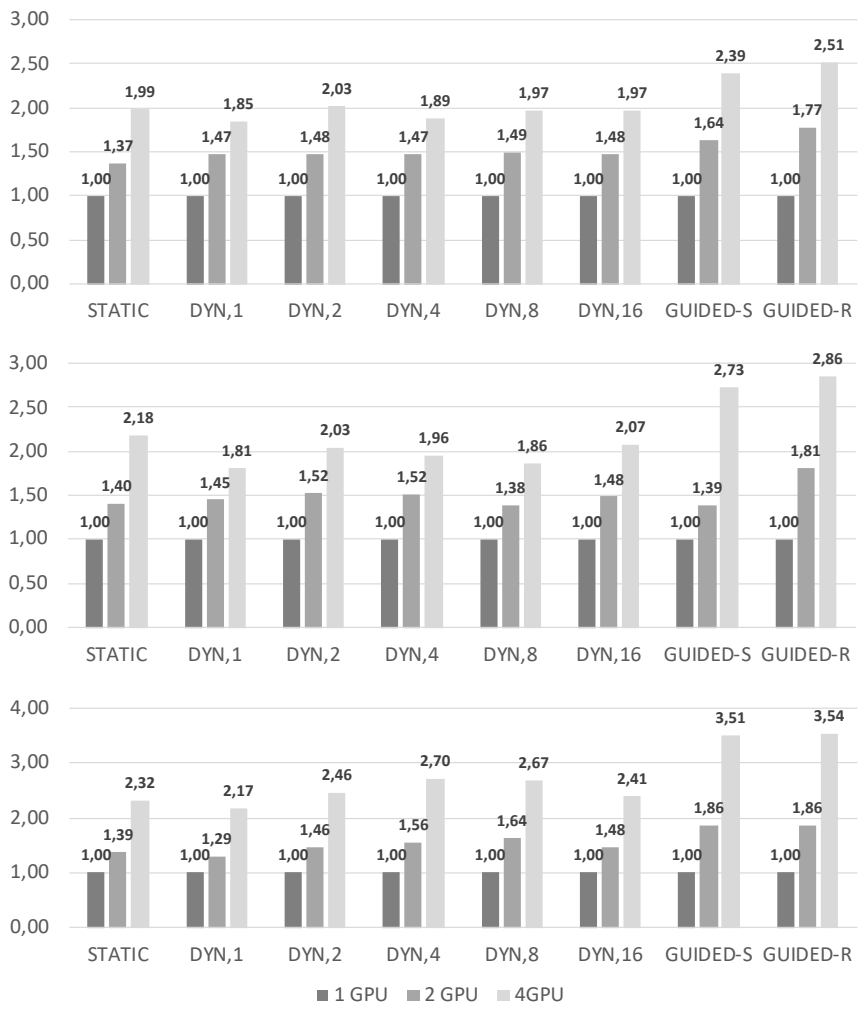

Fig. 12. Overall speedups achieved by the 2-GPU and 4-GPU executions with respect to the uni-GPU execution for BT-MZ application with all schedulers and input classes

from any significant slowdown in the Communication Period, it suffers from work unbalance.

For classes $\mathrm{C}$ and $\mathrm{D}$ these trends are even more emphasized. For class C, Guided schedulers outperform uniGPU execution by $2.73 x$ (Guided) and 2.99x (Sizes) with 4 GPUs. And in class D the speedups are $3.51 \mathrm{x}$ and $3.54 \mathrm{x}$ respectively, while the remaining schedulers outperform uni-GPU execution by less than $3 x$.

In conclusion, Static and Guided schedulers generate the best zone-to-GPU mapping for the execution of the Communication Period. Dynamic schedulers result in a zone placement where adjacency is not taken into account leading to excessive communication between the GPUs. But only the Guided schedulers offer a zone-to-GPU mapping that also solves the work unbalance problem in the Compute Period.

\section{Guided scheduling versus Optimal scheduling}

Finally, we study the performance lost caused by our guided scheduler with respect to the optimal scheduler (section 3.3.1). We have modified the BT-MZ application so that the zone-gpu mapping determined by the optimal scheduler is read from a file at the beginning of the execution of the application and it is applied to the Compute Period.

For class B the optimal scheduler results in an overall speedup of $2.58 \mathrm{x}$ with respect uni-GPU execution. Our proposed Guided scheduler performs at similar level (2.51x, just $3 \%$ below the optimal), having almost the same effect in the Compute Period and Communication Period as the optimal scheduler. The results for class $C$ expose the same situation, with a 2.91x speedup, better than the Guided scheduler (2.86x, just $2 \%$ below the optimal).

For class D, from the left side of Fig. 11, we can observe that the larger the class, the larger the weight of he Compute Period. Then, as the main difference between the optimal scheduler and the guided scheduler is the ability of the former to reduce Communication Costs, we expect that the larger the class, the smaller the difference between the execution times observed by the guided and the optimal schedulers. The results observed in classes B and C corroborate this hypothesis.

In general, the zone-gpu mappings obtained by both schedulers perform similarly. The cuts across the 2D mesh success on maximizing adjacency while keeping the work balanced in the Compute Period. In accordance, the resulting execution times are at similar level.

\begin{tabular}{|c|c|c|c|c|c|c|c|c|c|c|}
\hline & \multirow{2}{*}{ LU-MZ } & \multicolumn{3}{|c|}{ CLASS B } & \multicolumn{3}{|c|}{ CLASS C } & \multicolumn{3}{|c|}{ CLASS D } \\
\hline & & $1 \mathrm{GPU}$ & $2 \mathrm{GPU}$ & $4 \mathrm{GPU}$ & $1 \mathrm{GPU}$ & $2 \mathrm{GPU}$ & $4 \mathrm{GPU}$ & $1 \mathrm{GPU}$ & $2 \mathrm{GPU}$ & $4 \mathrm{GPU}$ \\
\hline \multirow{2}{*}{ Time } & Compute & 26,95 & 13,45 & 6,80 & 49,80 & 24,95 & 12,53 & 402,70 & 203,13 & 102,10 \\
\hline & Communication & 1,25 & 2,12 & 3,88 & 1,28 & 4,00 & 9,65 & 1,70 & 39,16 & 121,76 \\
\hline \multirow{2}{*}{ Speedup } & Compute & 1,00 & 2,00 & 3,96 & 1,00 & 2,00 & 3,97 & 1,00 & 1,98 & 3,94 \\
\hline & Communication & 1,00 & 0,59 & 0,32 & 1,00 & 0,32 & 0,13 & 1,00 & 0,04 & 0,01 \\
\hline \multirow{2}{*}{\multicolumn{2}{|c|}{ SP-MZ }} & \multicolumn{3}{|c|}{ CLASS B } & \multicolumn{3}{|c|}{ CLASS C } & \multicolumn{3}{|c|}{ CLASS D } \\
\hline & & $1 \mathrm{GPU}$ & $2 \mathrm{GPU}$ & $4 \mathrm{GPU}$ & $1 \mathrm{GPU}$ & $2 \mathrm{GPU}$ & $4 \mathrm{GPU}$ & $1 \mathrm{GPU}$ & $2 \mathrm{GPU}$ & $4 \mathrm{GPU}$ \\
\hline \multirow{2}{*}{ Time } & Compute & 9,33 & 4,69 & 2,38 & 36,90 & 18,62 & 9,34 & 433,50 & 216,50 & 109,00 \\
\hline & Communication & 1,56 & 2,40 & 3,77 & 6,50 & 8,18 & 10,96 & 28,40 & 35,40 & 49,80 \\
\hline \multirow{2}{*}{ Speedup } & Compute & 1,00 & 1,99 & 3,92 & 1,00 & 1,98 & 3,95 & 1,00 & 2,00 & 3,98 \\
\hline & Communication & 1,00 & 0,65 & 0,41 & 1,00 & 0,79 & 0,59 & 1,00 & 0,80 & 0,57 \\
\hline
\end{tabular}

Overall execution time (ms) and speedup of multi-GPU LU-MZ and SP-MZ with respect to uni-GPU. Static scheduler.

\subsubsection{LU-MZ benchmark}

LU-MZ operates over a grid of 16 zones, all of them equally sized. Therefore, the Static scheduling perfectly balances the Compute Period over the GPUs because it assigns the same number of zones to each GPU. Consequently, only the Static scheduling is studied for this application, without including a comparison with Dynamic and Guided schedulers.

\section{Compute Period vs Communication Period}

TABLE 2 shows the execution times and speed ups for the Compute Period and Communication Period from where we derive their relative contribution to the overall execution time. In class B, the Compute Period dominates the execution with $95 \%, 82 \%$ and $61 \%$ of the execution time with 1,2 and 4 GPUs respectively; this represents $26.95 \mathrm{~ms}, 13.45 \mathrm{~ms}$ and $6.80 \mathrm{~ms}$ respectively. The Communication Period takes $1.25 \mathrm{~ms}(5 \%), 2.12 \mathrm{~ms}(18 \%)$ and $3.88 \mathrm{~ms}(39 \%)(1,2$ and 4 GPUs respectively). Notice how the Compute Period is able to exploit the increment of GPUs, while the Communication Period suffers from a significant slowdown.

The Compute Period is speeded-up by $2 \mathrm{x}$ and 3.96x ( 2 and 4 GPUs respectively), the Communication Period degrades to $0.59 \mathrm{x}$ and $0.32 \mathrm{x}$ respectively. The slowdown is due to the appearance of zone transfers while computing the boundary values. Increasing the number of GPUs incurs into a zone distribution among them, generating additional zone transfers between the GPUs. Also, note that this period has no inter-zone parallelism, so increasing the number of GPUs does not reduce its execution time. 
Classes C and D expose exactly the same trend in terms of speedup of the Compute Period and slowdown of the Communication Period. For class C, speedup for the Compute Period is $2 \mathrm{x}$ and 3.97x (2 and 4 GPUs); Communication Period performance degrades to $0.32 x$ and $0.13 x$ respectively.

The slowdown is more significant in class $C$ than in class $B$ because class- $C$ zones are larger than class- $B$ zones, so the amount of data to be transferred between the GPUs increases in class $C$ respect to class $B$. Consequently, the slowdown observed using input class $\mathrm{D}$ is even worse. The class-D Communication Period takes 1.70 ms (1 GPU), 39.16 ms (2 GPUs), and $121.76 \mathrm{~ms}$ (4 GPUs); i.e., the performance degrades to $0.04 x$ and $0.01 x$ ( 2 and 4 GPUs respectively).

In conclusion, the scalability of LU-MZ application is heavily affected by a bottleneck in the Communication Period. The bigger the input class, the more data has to be transferred between the GPUs, and the slower the Communication Period. In contrast, the Compute Period presents an almost linear scalability in all of the tested input classes.

\section{Overall Performance}

Using 2 GPUs, all of the tested input classes outperform uniGPU execution (by 1.85x, 1.77x and 1.64x for classes B, C and $\mathrm{D}$ respectively). The bigger the input class, the smaller the speedup due to the performance degradation observed in the Communication Period. Using 4 GPUs the trend is even more pronounced: the observed speedups are $2.61 \mathrm{x}, 2.31 \mathrm{x}$ and $1.80 x$ for classes $B, C$ and $D$ respectively.

\subsubsection{SP-MZ benchmark}

SP-MZ operates over a grid with a number of zones that is a power of two, all of them equally sized. Therefore, the Static scheduler is able to perfectly balance the workload of the Compute Period among 2 of 4 GPUs because this scheduler distributes the same number of zones per GPU. Consequently, the Static scheduling is the only scheduler studied for this application.

SP-MZ application behaves like LU-MZ application. TABLE 2 shows the overall execution times and speedup achieved by our multi-GPU parallelization of SP-MZ. We avoid repeating similar explanations for the SP-MZ, and it has been kept in the paper to cover the whole set of applications in the NPB-MZ suite.

\section{Related Works}

The NAS Parallel Benchmarks (NPB) [5] is a suite of kernels and applications representative of most parallel workloads. NPB has become a de-facto standard in parallel-computing evaluation. There is a plethora of NPB implementations, but first implementations just exploited fine-grain parallelism.

Van der Wijngaart and Jin [6] extended the NPB applications (LU, BT and SP) to exhibit coarse-grain parallelism. As these applications operate on a three-dimensional mesh, the authors slice the mesh by two dimensions. Then, each resulting block (zone) can be solved independently; however, before computing the next time step, boundary values must be exchanged between neighbor zones. Their implementations, NPB Multi-Zone (NPB-MZ), exhibit both fineand coarse-grain parallelism. They developed two hybrid implementations (MPI+OpenMP and SMP+OpenMP) [11] for each application. Their results reveal good scalability and point out the impact of load balancing on the performance of BT-MZ, which zones are non-uniformly sized.

Dümmler and Rü nger [7] evaluated NPB-MZ benchmarks on hybrid CPU+GPU architectures. They decompose the workloads and, using a static scheduling, distribute them among the CPU's or the GPU. Their evaluations show a significant performance improvement with respect to both pure GPU and pure CPU implementations.

Pennycook et al. [12] detail their implementation of the LU-NPB application on CUDA. Moreover, they developed an analytical model to estimate the execution time of the benchmark on a range of architectures. They validate the model using evaluation environments that range from a single GPU to a cluster of GPU's. Their conclusions point out the implementation challenges on current architectures and the performance trends for future architectures.

$\mathrm{Xu}$ et al. [13] focused on directive-based parallelization of NPB benchmarks. After analyzing and profiling the OpenMP version of NPB, they annotate the source code with OpenACC directives to automatically generate GPU versions of the benchmarks. Their evaluations show performance results close to hand-tuned programs. Finally, they propose the addition of new annotation clauses to OpenACC in order to expose more parallelism.

Cabezas et al. [14] developed a programming framework and a runtime that is able to automatically distribute a kernel programmed for a single GPU across several GPUs. Their evaluations on several dense scientific computations reveal almost linear speedups.

Komoda et al. [15] developed an OpenACC compiler able to execute single-GPU OpenACC programs on multiGPU systems. They extended OpenACC standard by a few directives that allow expressing memory access patterns.

Loop scheduling has been studied for many type of applications and for both shared and distributed memory architectures. Adaptive loop schedulers have been proposed based on information gathered at runtime. In [8], [16], [17] loop schedulers combine information gathering (e.g.: runtime execution times or actual sizes of data structures) with the ability of memorizing the work assignment produced by the scheduler itself. Specifically for NAS-MZ benchmarks, [18] describes a feedback scheduler based on execution times to determine thread distribution and work assignment for NUMA shared memory architectures.

In [19], [20] loop schedulers address the work unbalance in irregular applications based on runtime information used to deploy dynamic schedulers. In [21] data dependencies across loop iterations are addressed and their information is gathered at runtime to design specific loop schedulers that maximize locality preserving the correct order of execution.

In [22], an on-line mechanism is presented that dedicates a single processor to execute a branch-and-bound algorithm to search for partial schedules concurrent with the execution of tasks previously assigned to the remaining processors.

Processor affinity to data associated to computations has been a essential factor for designing loop schedulers, targeting the preservation of data locality but minimizing the rations of work unbalance [23]-[25].

Task dynamic schedulers have been also studied, specially in the context of OpenMP programming model [26], 
[27]. They focus on the following issues: work balance, locality and cut-off mechanisms for divide-and-conquer algorithms which usually present implementations based on recursive iterative structures.

\section{Conclusions}

We have described and evaluated our multi-GPU implementation of the NPB-MZ benchmarks: it exposes both coarse-grain and fine-grain parallelism in order to be exploited by a multi-GPU system. As coarse-grain parallelism is distributed across GPUs, several work-distribution schemes (Static, Dynamic and Guided schedulers) have been studied to ensure a proper work balance. However, due to the nature of NPB-MZ benchmark suite, its execution alternates computational and communication phases. We have observed how the work-distribution schemes determine the data placement across the GPUs which in turn has a direct impact over the communications between the GPUs. Therefore, we have shown how the best schedulers are those that find the best trade-off between the computational and communication phases. Our evaluations show that Guided schedulers outperform all the other studied schedulers in finding this trade-off. The overall performance improvement with respect to single-GPU execution is from $1.48 x$ to $1.86 x$ ( 2 GPUs) and from $1.75 x$ to $3.54 x$ (4 GPUs).

\section{ACKNOWLEDGMENTS}

This work was supported by the Spanish Ministry of Science and Technology (TIN2015-65316-P) and by the Generalitat de Catalunya (2014-SGR-1051). We thank Professor Javier Larrosa for assistance with integer programming and the anonymous reviewers for their helpful comments.

\section{REFERENCES}

[1] M. A. et al., "Tensorflow: Large-scale machine learning on heterogeneous distributed systems," 2015. [Online]. Available: http://download.tensorflow.org/paper/whitepaper2015.pdf

[2] "Gpu-accelerated caffe." [Online]. Available: https://www.nvidia.com/en-gb/data-center/gpu-acceleratedapplications/caffe/

[3] S. Manavski and G. Valle, "Cuda compatible gpu cards as efficient hardware accelerators for smith-waterman string alignment," BMC bioinformatics, vol. 9 Suppl 2, p. S10, 022008.

[4] G. Giuntoli, J. Grasset, A. Figueroa, C. Moulinec, M. Vázquez, G. Houzeaux, S. Longshaw, and S. Oller, "Hybrid cpu/gpu fe2 multi-scale implementation coupling alya and micropp," 2019.

[5] D. Bailey, E. Barszcz, J. Barton, D. Browning, R. Carter, L. Dagum, R. Fatoohi, P. Frederickson, T. Lasinski, R. Schreiber, H. Simon, V. Venkatakrishnan, and S. Weeratunga, "The nas parallel benchmarks," Int. J. High Perform. Comput. Appl., 5(3), pp. 63-73, 1991.

[6] R. F. V. der Wijngaart and H. Jin, "Nas parallel benchmarks, multizone versions," NASA Ames Research Center, Tech. Rep. NAS-03010, Jul 2003.

[7] J. Dümmler and G. Rünger, "Execution schemes for the NPBMZ benchmarks on hybrid architectures: A comparative study," in Procs. of the Intl. Conf. on Parallel Computing, ParCo 2013.

[8] J. M. Bull, "Feedback guided dynamic loop scheduling: Algorithms and experiments," in Euro-Par'98 Parallel Processing, D. Pritchard and J. Reeve, Eds., 1998, pp. 377-382.

[9] G. L. Nemhauser and L. A. Wolsey, Integer and Combinatorial Optimization. USA: Wiley-Interscience, 1988.

[10] "IBM CPLEX Optimizer." [Online]. Available: https://www.ibm.com/analytics/cplex-optimizer

[11] H. Jin and R. F. V. der Wijngaart, "Performance characteristics of the multi-zone NAS parallel benchmarks," in 18th International Parallel and Distributed Processing Symposium, 2004, pp. 26-30.
[12] S. J. Pennycook, S. D. Hammond, S. A. Jarvis, and G. R. Mudalige, "Performance analysis of a hybrid MPI/CUDA implementation of the NASLU benchmark," SIGMETRICS Performance Evaluation Review, vol. 38, no. 4, pp. 23-29, 2011.

[13] R. Xu, X. Tian, S. Chandrasekaran, Y. Yan, and B. M. Chapman, "NAS parallel benchmarks for gpgpus using a directive-based programming model," in Languages and Compilers for Parallel Computing, J. C. Brodman and P. Tu, Eds., 2014, pp. 67-81.

[14] J. Cabezas, L. Vilanova, I. Gelado, T. B. Jablin, N. Navarro, and W.$\mathrm{m}$. Hwu, "Automatic parallelization of kernels in shared-memory multi-gpu nodes," in Intl. Conf. on Supercomputing, 2015, pp. 3-13.

[15] T. Komoda, S. Miwa, H. Nakamura, and N. Maruyama, "Integrating multi-gpu execution in an openacc compiler," in Procs. of the 2013 42nd Intl. Conference on Parallel Processing, 2013, pp. 260-269.

[16] C. D. Polychronopoulos and D. J. Kuck, "Guided self-scheduling: A practical scheduling scheme for parallel supercomputers," IEEE Transactions on Computers, vol. C-36, no. 12, pp. 1425-1439, 1987.

[17] Yong Yan, Canming Jin, and Xiaodong Zhang, "Adaptively scheduling parallel loops in distributed shared-memory systems, 8 (1)," IEEE Trans. on Parallel and Distributed Systems, vol. 8, no. 1 pp. 70-81, 1997.

[18] A. Duran, M. Gonzàlez, and J. Corbalán, "Automatic thread distribution for nested parallelism in openmp," in Proceedings of the 19th Annual International Conference on Supercomputing, 2005, p. 121-130.

[19] S. Lucco, "A dynamic scheduling method for irregular parallel programs," in Proceedings of the ACM SIGPLAN 1992 Conference on Programming Language Design and Implementation, 1992, p. 200-211.

[20] T. H. Tzen and L. M. Ni, "Trapezoid self-scheduling: a practical scheduling scheme for parallel compilers," IEEE Transactions on Parallel and Distributed Systems, vol. 4, no. 1, pp. 87-98, 1993.

[21] M. Gonzalez, E. Ayguade, X. Martorell, and J. Labarta, "Exploiting pipelined executions in openmp," in 2003 International Conference on Parallel Processing, 2003. Proceedings., 2003, pp. 153-160.

[22] B. Hamidzadeh and D. J. Lilja, "Self-adjusting scheduling: An on-line optimization technique for locality management and load balancing," in Intl. Conf. on Parallel Processing, 1994, p. 39-46.

[23] E. P. Markatos and T. J. LeBlanc, "Using processor affinity in loop scheduling on shared-memory multiprocessors," IEEE Transactions on Parallel and Distributed Systems, vol. 5, no. 4, pp. 379-400, 1994.

[24] S. Subramaniam and D. L. Eager, "Affinity scheduling of unbalanced workloads," in Supercomputing '94:Proceedings of the 1994 ACM/IEEE Conference on Supercomputing, 1994, pp. 214-226.

[25] E. Markatos and T. LeBlanc, "Load balancing vs. locality management in shared-memory multiprocessors," Tech. Rep., 1991.

[26] S. L. Olivier, A. K. Porterfield, K. B. Wheeler, M. Spiegel, and J. F. Prins, "Openmp task scheduling strategies for multicore numa systems," Int. J. High Perform. Comput. Appl. 26(2), p. 110-124, 2012.

[27] J. LaGrone, A. Aribuki, C. Addison, and B. Chapman, "A runtime implementation of openmp tasks," in International Workshop on OpenMP. Springer, 2011, pp. 165-178.

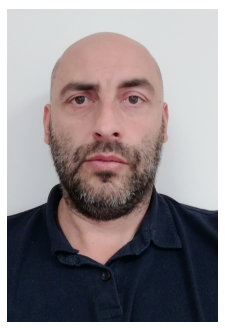

Marc Gonzalez Tallada received the degree in computer science in 1996 and the $\mathrm{PhD}$ degree in computer science in 2003, both from the Universitat Politècnica de Catalunya (UPC), Spain. In 2001, he joined the Department of Computer Architecture at UPC, where he is currently an associate professor. His research interests are related to programming models and compilers for High Performance Computing technologies.

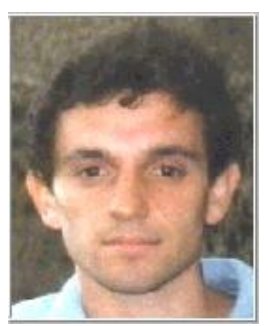

Enric Morancho received the degree in computer science in 1992 and the PhD degree in computer science in 2002, both from the Universitat Politècnica de Catalunya (UPC) ,Spain In 1993, he joined the Department of Computer Architecture at UPC, where he is currently an associate professor. His research interests include processor micro-architecture, memory hierarchy, awareness of architecture in programming and operating systems. 